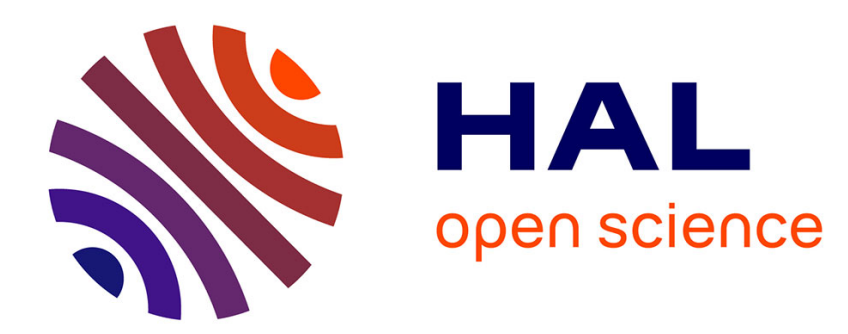

\title{
A 2D numerical study of the effect of particle shape and orientation on resistivity in shallow formations
}

\author{
Etienne Rey, Denis Jongmans
}

\section{To cite this version:}

Etienne Rey, Denis Jongmans. A 2D numerical study of the effect of particle shape and orientation on resistivity in shallow formations. Geophysics, 2007, 72 (1), pp.F9-F17. 10.1190/1.2399453 . hal00196175

\section{HAL Id: hal-00196175 \\ https://hal.science/hal-00196175}

Submitted on 12 Dec 2007

HAL is a multi-disciplinary open access archive for the deposit and dissemination of scientific research documents, whether they are published or not. The documents may come from teaching and research institutions in France or abroad, or from public or private research centers.
L'archive ouverte pluridisciplinaire HAL, est destinée au dépôt et à la diffusion de documents scientifiques de niveau recherche, publiés ou non, émanant des établissements d'enseignement et de recherche français ou étrangers, des laboratoires publics ou privés. 


\title{
A 2D NUMERICAL STUDY OF THE EFFECT OF PARTICLE SHAPE AND ORIENTATION ON RESISTIVITY IN SHALLOW FORMATIONS
}

\author{
Etienne Rey and Denis Jongmans \\ L.I.R.I.G.M., Grenoble University,BP 53X, 38041 Grenoble Cedex 9, France
}

Abstract

Surficial heterogeneous soils such as till, alluvial fans or slope deposits are difficult to characterize by geotechnical tests due to the presence of decimeter to meter sized pebbles or rocks. The effective resistivity of such two-component medium composed of a percentage of resistive particles embedded in a conductive matrix is given by the Bussian's equation. The application of this equation allows the concentration of resistive particles to be determined if the resistivity values of each component and of the mixture, as well as the cementation exponent $m$, are known. However, previous theoretical and experimental studies have shown that the effective resistivity is affected by the shape of the particles. The objective of this study is to numerically determine the $2 \mathrm{D}$ effects of particle shape and orientation on the resistivity. Two configurations have been considered in the Finite Element modeling: laboratory like measurements and field layout. For circular particles, the numerical results fit the Bussian's equation with an exponent $m$ of 2 . Aligned elongated particles induce an anisotropy which can raise or diminish the exponent $m$, depending on the particle orientation and on the tortuosity of the current paths. Field experiment simulations showed that $m$ varies 
between 2.5 and 3.1 for an aspect ratio of 5 and that anisotropy resulting from the particle shape has little effect ( $m$ close to 2 ) when this ratio is lower than 2.5. This increase of $m$ with the aspect ratio is in agreement with the theoretical model of Mendelson and Cohen and is consistent with the results of experimental studies. For laboratory measurement simulations, $m$ values vary between 1.3 and 4 for a particle aspect ratio of 5, whatever the resistivity contrast between the particles and the matrix. The difference of results between the two configurations is explained by the paradox of anisotropy.

Keywords : electrical resistivity, heterogeneous soil, anisotropy, particle shape, cementation exponent.

\section{Introduction}

Electrical prospecting methods are increasingly used for characterizing shallow geological layers. With the development of multi-electrode arrays and rapid inversion methods (e.g. Oldenburg and Li, 1994; Loke and Barker, 1996), ERT (Electrical Resistivity Tomography) emerged as a major tool for near-surface investigation purposes. Among others, common environmental and engineering applications are fluid migration (Park, 1998), detection of contaminants (Godio and Nadi, 2003), mapping of internal landfill structures (Bernstone et al., 2000), imaging faults (Demanet et al., 2001), landslide investigations (Havenith et al., 2000) and imaging of engineered hydraulic barriers (Daily and Ramirez, 2000). 
One of the major reasons for using electrical resistivity is the broad range of resistivity values (Reynolds, 1997), allowing the potential discrimination between various geological materials or their conditions, including water content, fracturing or contamination. Resistivity measurements have been widely used to assess various properties of geological formations, particularly by the oil industry for evaluating the porosity and hydrocarbon bearing capacity in sedimentary rocks. The quantitative interpretation of resistivity measurements is usually based on the experimental law (Archie, 1942), which for a porous saturated rock is:

$$
\rho_{0}=a \rho_{f} \phi^{-m}
$$

where $\rho_{0}$ is the resistivity of the rock, $\rho_{f}$ is the resistivity of the fluid, $\phi$ is the porosity (volume fraction of water filled pores) and $a$ and $m$ are two coefficients. The coefficient $m$ is often called the cementation exponent. Numerous studies (for a review, see Jackson et al., 1978; Chinh, 2000 or Friedman, 2005) have shown that a wide variety of unconsolidated sediments obey Archie's law with an exponent $m$ ranging between 1.3 and 4 and with a value of $a$ close to 1 . The coefficient $a$ must be strictly equal to 1 to satisfy the condition $\rho_{0}=\rho_{f}$ when $\phi=1$. Although $a=1$ is found to hold for clean sands, many core measurements require $a$ $\neq 1$ in equation 1 to obtain satisfactory fitting (Bussian, 1983). The presence of clays is then usually considered by adding a term explicitly incorporating clay conductivity in equation 1 , expressed in terms of conductivity, and by keeping $a=1$ (McNeill, 1990). Jackson et al. (1978) experimentally studied the resistivity-porosity particle relationship for marine sands and found that the exponent $m$ is mainly dependent on the shape of the particles, increasing from 1.2 for spheres to 1.9 for platey fragments.

In 1981, Sen et al. proposed a self similar model based on the Hanai-Bruggeman approach (Bruggeman, 1935; Hanai, 1968). In this model, the porous medium is characterized 
as a water suspension of solid grains and each rock grain is coated with a skin made of other coated grains. In the dc limit, the rock resistivity for non-conducting spherical particles is given by equation 1 with $a=1$ and $m=1.5$. For non spherical particles, the exponent $m$ is greater than 1.5 for platey grains and cylindrical particles with axis perpendicular to the electrical field, and smaller than 1.5 for plates and cylindrical particles with axis parallel to the external field.

Mendelson and Cohen (1982) and Sen (1984) generalized the theory of Sen et al. (1981) to the case of ellipsoidal particles with two distributions of orientations: randomly oriented particles in $3 \mathrm{D}$ and particles with aligned principal axis in $2 \mathrm{D}$. In the former case, the resistivity obeys Archie's law with $a=1$ and $m=1.5$ for spherical grains. The presence of oblate particles raises $m$ significantly. Aspect ratios of about 4, 10 and 14 increase the exponent $m$ to 2, 3 and 4, respectively. For 2D aligned particles, the resistivity is a tensor with principal values satisfying a generalized Archie's law:

$$
\rho_{i}=a_{i}(\phi) \rho_{f} \phi^{-m}
$$

where $\rho_{i}$ is the $i^{\text {th }}$ principal value of resistivity and $a_{i}(\Phi)$ is a porosity dependent coefficient. Mendelson and Cohen (1982) found that the exponent $m$ had a minimum value of 2 , independent of the particle shapes if all particles were aligned and had the same shape (including circular ones). Nevertheless, the coefficients $a_{i}$ in equation (2) depend on the particle shape and orientation, varying between 0.1 and 4 for grains with eccentricities in the range $0-0.95$. In the case of aligned particles with a distribution of shapes or if the particles are randomly oriented, the value of $m$ is increased. An output of the work of Mendelson and Cohen (1982) is that the rock resistivity is more sensitive to the porosity in $2 \mathrm{D}(m=2$ for circular grains) than in 3D ( $m=1.5$ for spherical grains). 
Bussian (1983) extended the work of Sen et al. (1981) to include a conducting rock matrix. Based on the Hanai-Bruggeman approach, he related the electrical properties of any heterogeneous two-component mixture to the properties of individual components. The effective resistivity $\rho_{0}$ of a biphasic medium composed of resistive particles $\left(\rho_{i}\right)$ embedded in a conductive matrix $\left(\rho_{f}\right)$ is given by:

$$
\frac{\rho_{0}}{\rho_{f}}=(1-C)^{-m}\left(\frac{1-\frac{\rho_{f}}{\rho_{i}}}{1-\frac{\rho_{0}}{\rho_{i}}}\right)^{-m}
$$

where $\mathrm{C}$ is the fractional volume of dispersed particles. This model is applicable to any formation in which a finite resistivity value can be ascribed to each component. It is worth noting that $\rho_{0}=\rho_{i}$ when $C=1$ and that $\rho_{0}=\rho_{f}$ when $C=0$. For very high resistivity contrasts $\rho_{i} / \rho_{f}$, equation 3 is similar to Archie's law (equation 1) with $a=1$.

All the above relationships, initially developed for oil industry, have applications in other areas, like in engineering geology where heterogeneous materials are commonly encountered. Figure $1 a$ shows a typical surficial alluvial fan deposit which is frequently found in the mountain ranges and especially in the Alps. It is made of limestone particles and blocks $(\mathrm{cm}$ to $\mathrm{dm}$ size) embedded in a matrix of marly clay. Particle size distribution (Figure $1 \mathrm{~b}$ ) shows the presence of a plateau for fine particle sizes and a strong gradient between 5 and $10 \mathrm{~mm}$, highlighting the two-component characteristic of the material (Figure 1c). The electrical structure of such deposit, made of resistive particles in a conductive matrix, is very similar to the one of a porous medium with resistive grains saturated with a conductive fluid. Recently, Rey et al. (2006) proposed to use equation 3 with $m=1.5 / 2$ in a $3 \mathrm{D} / 2 \mathrm{D}$ system for 
determining the concentration of stony particles in a coarse soil from in situ resistivity measurements on the deposit and on its two components. The use of these $m$ values is supported by experimental data on sphere-like and cylindrical inclusions. Several authors (among others, Atkins and Smith, 1961, Jackson et al., 1978, Friedman and Robinson, 2002) made experiments on natural sands and found $m$ values close to 1.5 for reported sphericity values ranging from 0.73 to 0.83 . Their results compare well with the Bussian's predictions for non-conducting particles and $m$ values of 1.5 (Figure 2). In 2D, Lobb and Forester (1987) studied the resistance of square metal sheets as a function of the area of randomly drilled holes. Their results (Figure 2) agree very well with the Bussian's prediction for $m=2$, up to a hole concentration of $40 \%$. Above this value, resistivity measurements diverge from the theoretical curve, probably as a result of the effect of overlapping holes (Lobb and Forester, 1987).

Shallow heterogeneous materials often show a preferential oriented fabric, such as till (Lawson, 1979; Rappol, 1985; Benn, 1995), colluvium (Millar and Nelson, 2001) or mudflow (Lindsay, 1968). The limestone particles shown in Figure la are not spherical and exhibit aspect ratio values as high as 2.5. If non-spherical (oblate or prolate) particles have some preferential alignment, the soil becomes anisotropic and its resistivity depends on the direction in which it is measured. Several authors examined the effect of particle shape and/or orientation on the electrical properties of the material, through laboratory investigation (Willye, 1953; Atkins and Smith, 1961; Jackson et al., 1978; Kujiper et al., 1996; Friedman and Robinson, 2002) or theoretical models (Sen et al., 1981; Mendelson and Cohen, 1982; Bussian, 1983; Kuijper et al., 1996; Zimmerman, 1996). About the influence of the particle shape, the main conclusion is that the mixture resistivity generally increases when particles are elongated, for both $2 \mathrm{D}$ or $3 \mathrm{D}$, leading to higher values of the cementation exponent $m$. As mentioned before, theoretical works highlighted that $m$ is higher in $2 \mathrm{D}$ than in $3 \mathrm{D}$, showing the higher sensitivity of resistivity to porosity (or particle concentration) in 2D. Experimental 
results for non-spherical particles are plotted in Figure 2 and compared to the predictions of the Bussian's equation for non-conducting particles and for three values of the cementation exponent $(m=1.5,2$ and 3). Atkins and Smith (1961) showed that $m$ became greater as the particles became more platy: 1.6 for sand, 1.9 for kaolinite, 2.7 for calcium montmorillonite and 3.3 for sodium montmorillonite (Figure 2). Another experimental work on the relationships between particle shape and resistivity was made by Jackson et al. (1978). They showed that samples of natural sands have values of $m$ in the range 1.4 (round sand, sphericity of 0.83 ) to 1.6 (platy sand, sphericity of 0.78 ), consistently with the theoretical value (1.5) for spherical grains. For shell fragments (sphericity of 0.5 ) they found $m=1.9$. Recently, Friedman and Robinson (2002) made electrical measurements on quartz sand and tuff grains with a mean sphericity of 0.73 and 0.59 , respectively. The obtained resistivity values are slightly lower and higher than the ones expected for spherical particles (Figure 2). Jackson et al. (1978) linked these $m$ variations with sphericity to the increase of the tortuosity of the current flow paths. To our knowledge, no systematic experimental work addressed the influence of the particle orientation on the mixture resistivity. For aspect ratios ranging from 1/5 (oblate shape) to 5 (prolate shape), Kuijper et al. (1996) calculated the anisotropy in the conductivity parallel and perpendicular to the principal axis for packings of aligned non-conducting ellipsoids, using a random walk algorithm. They found significant variations of the cementation exponent $m$ with the aspect ratio and the component of the conductivity tensor. For aligned oblate ellipsoids and a porosity of 0.5 , they determined $m$ values of 1.2 and 2.6 for particles being respectively parallel or perpendicular to the current flow. In all previous studies the particles were assumed to be non-conducting.

This paper aims at studying the influence of the shape and orientation of finite resistive particles on electrical measurements, using 2D numerical modeling. Two measurement configurations were considered in the modeling, corresponding to laboratory and field tests. The limitation to $2 \mathrm{D}$ computations is justified by the dense meshing and the large number of 
finite elements required for correctly simulating the current flow in a heterogeneous medium. The consistency of the numerical results with the existing theoretical models and experimental data will be analyzed and the extension of the results to the $3 \mathrm{D}$ case will be discussed.

\section{D Numerical modelling}

The effect of particle shape and orientation on the effective resistivity of a heterogeneous medium was simulated for laboratory or field measurements, as shown in Figure 3. Laboratory tests (Figure $3 a$ ) consist in applying a current $I$ through a core sample (Butler and Knight, 1995; Chan et al., 2000). In such a configuration, the surface current electrodes $\left(\mathrm{C}_{1}\right.$, $\mathrm{C}_{2}$ ) are in contact with the extremities of the sample and the induced difference of potential $\Delta V$ is measured between two electrodes $\left(\mathrm{P}_{1}, \mathrm{P}_{2}\right)$ along the sample surface. The apparent resistivity is given by equation 4 , where $\mathrm{P}_{1} \mathrm{P}_{2}$ is the distance between the potential electrodes and $A$ is the sample cross sectional area:

$$
\rho_{a}=\frac{A}{P_{1} P_{2}} \frac{\Delta V}{I}
$$

Electrical Resistivity Tomography (ERT) is now a classical and widely used multi electrode method for imaging the subsurface (Reynolds, 1997). Several layouts can be used for acquiring the data (for a recent review, see Dahlin and Zhou, 2004). In such in-situ measurements, a current $I$ is injected between two current electrodes $\left(\mathrm{C}_{1}, \mathrm{C}_{2}\right)$ and the difference of potential is measured between two potential electrodes $\left(\mathrm{P}_{1}, \mathrm{P}_{2}\right)$ (Figure $\left.3 b\right)$. In $2 \mathrm{D}$, the apparent resistivity $\rho_{a}$ for a configuration of four electrodes is given by equation 5 . 


$$
\rho_{a}=\frac{\Delta V}{I} \times \frac{\pi}{\ln \left(\frac{P_{1} C_{2} \times P_{2} C_{1}}{P_{1} C_{1} \times P_{2} C_{2}}\right)}
$$

One of the most common system is the Wenner- $\alpha$ layout using a collinear arrangement of four equally spaced electrodes (Barker, 1979). In numerical modeling we simulated a Wenner- $\alpha$ array with a maximal number of 50 electrodes, and for minimal and maximal electrode spacings $\left(a_{\min }, a_{\max }\right)$ of $8 R$ and $12 R$, respectively, where $R$ is the particle radius. The simulated sequence is composed of 68 measurements of apparent resistivity, representing half of the maximum number of possible measurements. Numerical tests showed that this reduction, which significantly decreases the computation time, does not affect the average apparent resistivity. In the case of an heterogeneous medium mixing a conductive matrix and resistive particles, the aim of these measurements is to obtain the effective resistivity value corresponding to the homogenized medium and then to deduce the particle concentration. For field measurements, some relations are required between the minimum electrode spacing $a_{\min }$ and the particle size $R$ to ensure the homogenization. The correct simulation of the current flow in a heterogeneous medium also imposes strong constrains on the geometric and numerical parameters, which are discussed below.

We used the finite-element (F.E.) code CESAR developed by the LCPC (Humbert, 1989; Mestat, 1997; Marescot et al., 2006) for simulating the current flow at steady state. The mesh is composed of three-node elements. The 2D model structure (Figure 4) is composed of a rectangular conductive body (resistivity $\rho_{M}$ ) containing randomly spread circular or elongated resistive particles (radius $R$, resistivity $\rho_{l}$ ). In the case of field simulations, this heterogeneous zone is bordered by an external homogeneous area (resistivity $\rho_{\mathrm{e}}$, Figure 4), whose effect on 
the simulated measurements was corrected (Rey, 2005). In a first step, the numerical and geometric conditions were extensively tested in order to insure the correctness of the FE results within the heterogeneous zone. Details on the meshing and geometric requirements can be found in Rey et al. (2006). Here, the main conditions controlling the number of elements are outlined: (1) the size of the model is constrained by the boundary conditions (null potential), a maximum error of $0.5 \%$ being imposed on the resistivity values. The mesh must then be respectively over $30 a_{\max }$ large and $15 a_{\max }$ deep, where $a_{\max }$ is the maximum electrode spacing in the Wenner- $\alpha$ array, (2) the number of finite elements around each particle must be over 40 to limit numerical error under $0.5 \%,(3)$ the ratio $(a / R)$ between the electrode spacing $a$ and the particle radius $R$ must be greater than 8 in order to homogenize the mixture of soil and particles (Rey et al., 2006), (4) the correct simulation of the current flow between two neighbor particles requires at least two finite elements, limiting the volume percentage of particles to a maximum of $40 \%$. For these conditions, the elemental sampling volume can be assessed to be 60 times to 100 times larger than the particle size for getting an effective medium representation.

The maximum number of finite elements in the code is limited to $1.10^{5}$. With this constrain and the above requirements, $2 \mathrm{D}$ simulations impose a number of finite elements close to this limit, making the correct $3 \mathrm{D}$ modeling of the problem not possible with this computational capacity.

\section{Numerical results}

For each numerical computation, ten models were randomly simulated in order to get the average resistivity and the error bars (two standard deviations). The influence of circular resistive particles randomly dispersed in a conductive matrix was first simulated for both 
laboratory and field measurements (Figure 3), using FE analysis with the conditions specified above. A contrast of 1000 between matrix and inclusions was considered, with concentrations $C$ ranging from $0 \%$ to $40 \%$ at increment of $5 \%$. For each concentration value, the average normalized resistivity values $\rho_{n}=\rho_{o} / \rho_{M}$ versus particle concentration are given in Figure $5 a$, with the error bars. As the electrode spacing was chosen to homogenize the mixture, the mean apparent resistivity can be considered as the effective resistivity of the medium (Rey, 2005). For a high resistivity contrast $\left(\rho_{i} / \rho_{M}=1000\right)$, Figure 5 a shows a resistivity augmentation due to the particle percentage and a progressive increase of the slope of the curve with concentration values. For circular particles, the normalized effective resistivity $\rho_{n}$ does not depend on the type of simulated measurements (laboratory or field) and reaches a value of 2.7 at a concentration of $40 \%$. In the same figure the main theoretical laws with the parameters corresponding to the simulated case are plotted. Equation 2 (Mendelson and Cohen, 1982) and equation 3 (Bussian, 1983) using $m=2$, both provide a good fit to the numerical results. This $m$ value was given by Mendelson and Cohen (1982) in the generalized Archie's law for two dimensions when the particles all have the same shape. The plot of the Bussian's equation for $m=1.5$ (spherical particles) in the same figure highlights its sensitivity to the exponent $m$. For such a high resistivity contrast (1000), the hypothesis of non conducting particles made by Mendelson and Cohen in equation 2 is quite satisfied, allowing a good description of numerical results. However, as shown further, the Bussian's equation offers the advantage to take into account a finite resistivity contrast. These results support the use of Bussian's equation with an exponent $m$ of 2 for explaining the 2D resistivity variations resulting from the presence of resistive particles in a conductive matrix. Moreover, numerical simulations performed on different particle size populations $\left(R, R^{\prime}=R / 2, R+R^{\prime}\right.$ in equal proportions) show that the effective resistivity of the medium is not or little influenced by the size scale of the inclusions (figure $5 b{ }_{3}$ experimentally found by Jackson et al.(1978) on natural sands. Nevertheless, as mentioned above, several authors have shown that the exponent $m$ is 
influenced by the particle shape and orientation. These effects are now studied in two dimensions using numerical modeling.

Non circular particles were defined by two secant arc circles giving a lens shape (Figure 6). This choice was made for the easiness to introduce such shape in the meshing. The particle shape is characterized by its aspect ratio $A r$ which is equal to the ratio of the long and short axes. Two aspect ratio values (2.5 and 5) were considered and the meshing of the three particle types (including the circular ones) is illustrated in Figure 6. The particles were randomly dispersed with a constant orientation of their long axis (horizontal, inclined at $45^{\circ}$ and vertical), introducing an anisotropy in the medium (Figure 7). Computations were performed in the same way as for the circular particles.

Laboratory measurements with a resistivity contrast $\rho_{i} / \rho_{M}=1000$ were simulated for the two aspect ratio values and the three orientations (Figure 8). For Ar=2.5 (Figure 8a), the effective resistivity for vertical particles (i.e. transverse to the current flow) is significantly higher $\left(\rho_{n}=3.7\right.$ for $C=40 \%$ ) than for circular ones $\left(\rho_{n}=2.7\right)$ and the difference increases with the particle concentration. On the contrary, horizontally elongated particles (i.e. parallel to the current flow) yield effective resistivity values lower $\left(\rho_{n}=2.1\right)$ than the ones obtained for circular ones. Finally, the effective resistivity for oblique particles is slightly higher than for circular ones. When the aspect ratio rises from 2.5 to 5 (Figure $8 b$ ), the effect on the effective resistivity is more significant than from 1 to 2.5 , with higher values for vertical $\left(\rho_{n}=6.2\right.$ for $C=40 \%)$ or inclined particles $\left(\rho_{n}=4.4\right)$, whereas the effective resistivity values are only slightly lowered $\left(\rho_{n}=1.9\right)$ for horizontal particles. As the resistivity contrast is high $\left(\rho_{i} / \rho_{M}=\right.$ 1000), numerical results for horizontal and vertical particles are compared in the same figure with the theoretical formula (Equation 2) given by Mendelson and Cohen (1982) in 2D for elliptic particles. The equation predicts similar resistivity variations with the particle orientation. A very good fit is obtained for particles parallel to the current flow (horizontal 
orientation) for the two aspect ratio values. When particles are transverse to the current flow (vertical orientation), the theoretical effective resistivity is higher than the one obtained from numerical simulations for a concentration over $20 \%$. This discrepancy is probably due to the difference of shape between a lens and an ellipse, lenses being thinner at their extremities than ellipses for an equivalent aspect ratio, and consequently more easily crossed by the current paths.

Field measurements simulated for the same characteristics (Figure 9) show that the particle orientation has a weak influence on the resistivity values for an aspect ratio value of 2.5, even for concentration values as high as $40 \%$. For $A r=5$ the effect of the particle orientation is more noticeable. Resistivity values for all orientations are higher than the ones for circular particles, the highest resistivity $\left(\rho_{n}=4.0\right.$ for $C=40 \%$ ) being obtained for horizontal particles. This result will be discussed later. For $A r=5$, the effective resistivity values are bracketed by Bussian's curves with an exponent of 2.5 and 3.1 while the maximal exponent is lower than 2.3 for $A r=2.5$. This increase of the exponent $m$ with the particle aspect ratio is consistent with the results from laboratory investigation (Jackson and al., 1978) and with theoretical models (Mendelson and Cohen, 1982). This effect of the introduced anisotropy, which results from the augmentation of the tortuosity within the medium, appears to become significant for aspect ratio higher than 2.5.

All the numerical results presented so far relate to a large contrast of resistivity between particles and matrix $\left(\rho_{i} / \rho_{M}=1000\right)$ and we now test the influence of this contrast on the effective resistivity. Figure 10 shows the evolution of the effective resistivity with the particle concentration for an aspect ratio $A r=5$ and three different values of electrical contrast $\left(\rho_{i} / \rho_{M}=\right.$ $10,100,1000)$. On this graph, the electrode configuration is the one for laboratory experiments (Figure 3a). As expected, the effect of anisotropy on the effective resistivity is less apparent when the contrast is low. However, the fitting of the numerical results with the Bussian's law yields the same range of $m$ (from 1.3 for horizontal particles to 4 for vertical 
ones) whatever the contrast of resistivity. The invariance of the $m$ exponent with the resistivity contrast has been also observed for field measurements simulations (not shown). These results stress out the relationship between the exponent $m$ and the tortuosity.

\section{Discussion}

The comparison between laboratory (Figure 8) and field (Figure 9) measurement simulations shows the higher sensitivity of the laboratory resistivity measurements to anisotropy. Another striking feature is that the maximum effective resistivity is obtained for two different orientations: vertical particles in laboratory measurements and horizontal ones in field measurements. This apparent discrepancy in the results is explained by the theory of electrical anisotropy (Keller and Frischknecht, 1966; Wait, 1990). Stratified rocks made of a succession of conductive and resistive layers show a two-dimensional anisotropy that can be defined by two variables: the longitudinal resistivity $\left(\rho_{L}\right)$ measured parallel to the plane of stratification and the transverse resistivity $\left(\rho_{T}\right)$ measured normal to this plane. As expected, $\rho_{L}$ is lower than $\rho_{T}$, and the coefficient of anisotropy, defined as $\lambda=\sqrt{ }\left(\rho_{T} / \rho_{L}\right)$, generally ranges between 1 (homogeneous isotropic medium) and 2 (Keller and Frischknecht, 1966). In the case of horizontal layers, the apparent resistivity $\rho_{a L}$ measured by an electrode array at the surface is equal to $\lambda \rho_{L}$. When the layers are vertical and the array is oriented perpendicularly to the bedding, $\rho_{a T}$ equals $\rho_{L}$. In other words, the apparent transverse resistivity $\rho_{a T}$ is then equal to the longitudinal resistivity $\rho_{L}$ and is lower than the apparent longitudinal resistivity $\rho_{a L}$.

This paradox of anisotropy is in accordance with the numerical modeling results in medium containing elongated aligned particles, as illustrated in Figure 11. Indeed, for laboratory measurement simulations (Figure 11a, Figure 11b), the longitudinal resistivity $\rho_{L}$ for horizontal particles (oriented parallel to the current lines) is lower than the resistivity $\rho_{T}$ for vertical particles (oriented transverse to the current lines). For field measurement 
simulations (Figure 11c, Figure 11d), the apparent resistivity for horizontal particles $\left(\rho_{a L}\right)$ is higher than the one $\left(\rho_{a T}\right)$ for vertical ones. As already evinced for stratified media (Watson and Barker, 1999), the apparent resistivity ellipses in a medium with elongated particles differ from the true ones (Figure 11). More quantitatively, we calculated the apparent resistivity values $\rho_{a T}$ and $\rho_{a L}$, considering the theory of electrical anisotropy and the $2 \mathrm{D}$ resistivity values $\rho_{L}$ and $\rho_{T}$ derived from equation 2. The obtained theoretical curves for an aspect ratio of 5 and an infinite resistivity contrast (Figure 12) are compared with the numerical results for 2D simulated field experiments (with a resistivity contrast of 1000). The fitting is very good for horizontally oriented particles. On the contrary, the theoretical curve is lower than the resistivity values for vertically aligned particles and for circular particles (Figure 12). These results suggest an influence of the tortuosity in the case of vertically aligned particles, which increases the resistivity values with regards to the theory of anisotropy.

For circular particles, the 2D numerical results presented in this paper are consistent with the Bussian's equation (with $m=2$ ) within a wide range of resistivity contrast (from 10 to 1000) and also with the experimental results of Lobb and Forrester (1987) in the $0-40 \%$ concentration range. They support the use of Bussian's equation as an extension of Archie's law for finite resistive particles. The numerical simulations for elongated aligned nonconducting particles are in agreement with the 2D model of Mendelson and Cohen (1982), highlighting the resistivity anisotropy resulting from the particle shape and orientation. For the maximum studied aspect ratio $(A r=5), m$ values obtained for current lines parallel and perpendicular to the principal ellipse axis vary from 1.3 to 4 , bracketing the value $(m=2)$ for circular particles. These $m$ values are higher than the ones obtained by Kuijper et al. (1996) in 3D for a porosity of 0.5 , i.e. $m=1.2$ and $m=2.6$ for aligned oblate ellipsoids with the same aspect ratio. This is in agreement with the increase of $m$ from 3D (1.5) to 2D (2) for spherical particles. Finally, 3D experimental data (Figure 2) on elongated particles with an aspect ratio close to 2.5 yield $m$ values around $1.8-1.9$, which are higher than 1.5 due to the tortuosity but 
lower than the corresponding ones computed from 2D numerical simulations ( $m$ about 2.3, Figure 9a). These results highlight the greater sensitivity of effective resistivity to particle concentration in the $2 \mathrm{D}$ case.

An interesting feature evinced by the laboratory measurements simulations is the apparent dependence of the cementation exponent $m$ with the concentration $C$, particularly for a high resistivity contrast and when the particles are perpendicular to the current lines (Figure $10 a$ ). For particles with an aspect ratio of $5, m$ increases from 3.6 for $C=0.4$ (porosi 0.6 ) to 5.2 for $C=0.1$ (porosity of 0.9 ). Such a variation was already found by Kuijper et al. (1996) for aligned oblate ellipsoids with the same aspect ratio, with an increase of $m$ from 2.6 to 3.7 for a porosity of 0.5 to 0.9 , respectively. These numerical results should be validated by experimental data in the future.

\section{Conclusions}

The effect of aligned elongated resistive particles embedded in a conductive matrix on the effective resistivity was studied by 2D FE modeling for two types of configurations: laboratory like experiments and field layouts. For circular particles, simulated effective resistivity values fit with the theoretical Bussian's equation with an exponent $m$ of 2 , whatever the resistivity contrast between the particles and the matrix. These results, along with the existing 2D experimental data, support the use of the Bussian's equation as an extension of Archie's law for finite resistive values. The size distribution of circular inclusions was found to have negligible influence on the effective resistivity.

In both configurations, the aspect ratio and alignment of particles induce an anisotropy which may generate significant variations on the measured effective resistivity. For field 
experiment simulations, numerical results evinced an increase of the effective resistivity and of the exponent $m$ with the aspect ratio, whatever the particle orientation and the resistivity contrast. Simulation results could always be well fitted with Bussian's equation, by increasing the exponent $m$ with the aspect ratio of the particles. For laboratory measurements, the effective resistivity for elongated particles can be lower or higher than the value for circular ones, depending on the angle between the current flow paths and the particle major axis. Accordingly, $m$ values for an aspect ratio $A r=5$ range between 1.3 for longitudinal orientation and about 4 for transversal one, bracketing $m=2$ (isotropic heterogeneous medium containing circular particles). The contrast of resistivity between matrix and particles was also found to have little influence on the exponent $m$. The difference of results between the two configurations is explained by the paradox of anisotropy. These numerical results compare well with the 2D model of Mendelson and Cohen (1982) for insulating particles. They also show $m$ variations with the particle orientation, similar to the ones obtained in 3D by Kuijper et al. (1996) but with a wider range. This illustrates the higher sensitivity of effective resistivity to anisotropy in the $2 \mathrm{D}$ case. Also, a decrease of the exponent $m$ with the particle concentration was observed, in agreement with the $3 \mathrm{D}$ numerical results of Kuijper et al. (1996). Our results might have applications in different fields in which two-component media including non-spherical particles are studied: petrophysics, composite materials, colloids and surficial heterogeneous soils (pebble fabrics in tills, alluvial fans). In such natural deposits however, aspect ratios are rarely higher than 2.5 , value for which the $2 \mathrm{D}$ anisotropy effect on field measurements is found to be weak. In $3 \mathrm{D}$, this effect is even less significant. Then, if the medium can be considered as biphasic, the effective electrical resistivity can be used as a robust parameter for estimating the resistive particle concentration in a wide range of surficial deposits.

\section{Acknowledgements}


This research was supported by the "Pôle Grenoblois des Risques Naturels".

\section{References}

Archie, G. E., 1942, The electrical resistivity $\log$ as an aid in determining some reservoir characteristics: Petr. Tech. 1, 55-62.

Atkins, E. R., and G. H. Smith, 1961, The significance of particle shape in formation factor porosity relationship: J. Petrol. Tech., 13, 285-291.

Barker, R., 1979, Signal contribution section and their use in resistivity studies: Geophysical Journal of the Royal Astronomical Society, 59, 123-129.

Benn, D. I., 1995, Fabric of subglacial till deformation, Breidamerkujökull, Iceland: Sedimentology, 42, 735-747.

Bernstone, C., T. Dahlin, W. Ohlsson and W. Hogland, 2000, DC-resistivity mapping of internal landfill structures: two pre-excavation surveys: Environmental Geology, 39, 360371.

Bruggeman, D. A. G., 1935, Berechnung verschidener physikalisher Konstanten von Heterogenen Substanzen: Annalen der Physik (Leipzig), 24, 636-679.

Bussian, A. E., 1983, Electrical conductance in a porous medium, Geophysics, 48, 12581268. 
Butler, D. B., and R. J. Knight, 1995, The effect of steam quality on the electrical behavior of steamflooded sands: Geophysics, 60, 998-1006.

Chan, C. Y., H. M. Buettner, N. Newmark and G. Mavko, 2000, Conductivity Measurements of sandy-clays mixtures: a modified experimental method: J. of Environmental and Engineering Geophysics, Vol. 5, Issue 3, 15-26.

Chinh, P. D., 2000, Electrical properties of sedimentary rocks having interconnected watersatured pore spaces: Geophysics, 65, 1093-1097.

Dahlin, T., and B. Zhou, 2004, A numerical comparison of 2D resistivity imaging with 10 electrode arrays: Geophysical Prospecting, 52, 379-398.

Daily, W., and A. L. Ramirez, 2000, Electrical imaging of engineered hydraulic barriers:

Geophysics, 65, 83-94.

Demanet, D., F. Renardy, K. Vanneste K., D. Jongmans, T. Camelbeeck, and M. Meghraoui, 2001, The use of geophysical prospecting for imaging active faults in the Roer graben, Belgium, Geophysics, 66, 78-89.

Friedman, S. P., and D. A. Robinson, 2002, Particle shape characterization using angle of repose measurements for predicting the effective permittivity and electrical conductivity of saturated granular media, Water Resources Research, 38, 1236-1246. 
Friedman, S. P., 2005, Soil properties influencing apparent electrical resistivity: a review: Computers and electronics in agriculture, 46, 45-70.

Godio, A., and M. Naldi, 2003, Two-dimensional electrical imaging for detection of hydrocarbon contaminants: Near Surface Geophysics, 1, 131-137.

Hanai, T., 1961, Dielectric theory on the interfacial polarization for two-phase mixtures: Bull. Inst. Chem. Res. Kyoto Univ., 39, 341-367.

Havenith, H.-B., D. Jongmans, K. Abdrakmatov , P. Tréfois, D. Delvaux, and I. A. Torgoev, 2000, Geophysical investigations on seismically induced surface effects, case study of a landslide in the Suusamyr valley, Kyrgyzstan: Survey of Geophysics, 21, 349-369.

Humbert, P., 1989, CESAR-LCPC : Un code général de calcul par éléments finis: Bulletin de Liaison des Laboratoires des Ponts et Chaussées, 160, 112-115.

Jackson, P. D., D. Taylor Smith, and P. N. Stanford, 1978, Resitivity-porosity-particle shape relationships for marine sands: Geophysics, 43, 1250-1268.

Keller, G. V., and F. C. Frischknetch, 1966, Electrical methods in geophysical prospecting: Pergamon press.

Kuijper, (de) A., R. K. J. Sandor, J. P. Hofman and J. A. de Wall, 1996, Conductivity of twocomponent systems: Geophysics, 61, 162-168. 
Lawson, D. E., 1979, A comparison of the pebble orientation in the deposits of the Matanuska Glacier, Alaska: Journal of Geology, 87, 629-645.

Lindsay, J. F., 1968, The development of clast fabric in mudflows: Journal of sedimentary petrology, 38, 1242-1253.

Lobb, C. J., and M. G. Forrester, 1987, Measurement of nonuniversal critical behavior in a two-dimensional continuum percolating system, Phys. Rev. B., 35, 1899-1901.

Loke, M. H., and R. D. Barker, 1996, Rapid least square inversion of apparent resistivity pseudosections by a quasi-Newton method: Geophysical Prospecting, 44, 131-152.

Marescot, L., S. Rigobert, S. Palma Lopes, R. Lagabrielle and D. Chapellier, 2006, A general approach for DC apparent resistivity evaluation on arbitrarily shaped 3D structures: J. of Applied Geophysics (in press).

McNeill, J. D., 1990, Use of Electromagnetic Methods for Groundwater Studies, in S. H. Ward, ed., Geotechnical and Environmental Geophysics, v. I: Tulsa, Oklahoma, Society of Exploration Geophysicists, 191-218.

Mendelson, K. S., and M. H. Cohen, 1982, The effect of grain anisotropy on the electrical properties of sedimentary rocks: Geophysics, 47, 257-263.

Mestat, P., 1997, Maillages d'éléments finis pour les ouvrages de géotechnique. Conseils et recommandations : Bulletin de liaison des Laboratoires des Ponts et Chaussées, 212, 3964. 
Millar, S. W. S., and F. E. Nelson, Sampling-surface orientation and clast macrofabric in periglacial colluvium: Earth Surface Processes and Landforms, 26, 523-529.

Oldenburg, D. W., and Y. Li, 1994, Inversion of induced polarization data: Geophysics, 59, $1327-1341$

Park, S., 1998, Fluid migration in the vadose zone from 3-D inversion of resistivity monitoring data, Geophysics, 63, 41-51.

Rappol, M., 1985, Clastic fabric strength in tills and debris flows compared for different environments: Geologie en Mijnbouw, 64, 327-332.

Rey, E., 2005, Caractérisation de sols hétérogènes par des méthodes géophysiques: Ph.D. thesis, University of Grenoble, France.

Rey, E., D. Jongmans, S. Garambois and P. Gotteland P., 2006, Characterisation of soils with stony particles using geoelectrical measurements: J. of Applied Geophysics, 58, 188-201.

Reynolds, J., 1997, An Introduction to applied and environmental geophysics: Wiley, John \& Sons, Inc.

Sen, P. N., C. Scala, and M. H. Cohen, 1981, A self-similar model for sedimentary rocks with application to the dielectric constant of fused glass beads: Geophysics, 46, 781-795. 
Sen, P. N., 1984, Grain shape effect on dielectric and electrical properties of rocks: Geophysics, 49, 586-587.

Wait, J. R., 1990, Current flow into a 3-dimensionnally anisotropic conductor: Radio Science, 25, 689-694.

Watson, K. H., and R. D. Barker, 1999, Differentiating anisotropy and lateral effects using azimuthal resistivity offset Wenner soundings: Geophysics, 64, 739-745.

Wyllie, M. R. J., and G. R. Gregory, 1953, Formation factor of unconsolidated porous media : influence of particle shape and effects of cementation: Petrol. Trans. AIME, 198, 103-110.

Zimmerman, R. W., 1996, Effective conductivity of a two-dimensional medium containing elliptical inclusions: Proceedings of the Royal Society, A452, 1713-1727.

Hugo Fricke, A mathematical treatment of the electric conductivity and capacity of disperse systems, Phys. Rev., 24, 575, (1924).

E.J. Garboczi and J. F. Douglas, Intrinsic conductivity of objects having arbitrary shape and conductivity, Phys. Rev. E, 53, 6169, (1996).

Lei Gao, Maxwell-Garnett type approximation for nonlinear composites with shape distribution, Phys. Lett. A, 309, 435, (2003)

S. Torquato, Random heterogeneous materials. Microstructure and macroscopic properties. Springer Verlag, (2002)

Paul W.J. Glover, Malcolm J. Hole, Jaume Pous, A modified Archie's law for two conducting phases, Earth and Planetary Science Letters, 180, (2000), 369-383

Scott B. Jones 1 and Shmulik P. Friedman, Particle shape effects on the effective permittivity of anisotropic or isotropic media consisting of aligned or randomly oriented ellipsoidal particles, WATER RESOURCES RESEARCH, VOL. 36, NO. 10, PAGES 2821-2833, (2000)

J. Kovacik, Electrical conductivity of two-phase composite material, Scripta Materialia, Vol. 39, No. 2, pp. 153-157, (1998) 


\section{Figure captions}

Fig. 1: (a) Picture of an alluvial fan deposit in the Alps. (b) Particle size distribution of the deposit shown in Figure 1a. (c) Representation of the deposit as a biphasic medium made of resistive particles (white circles) embedded in a conductive matrix.

Fig. 2: Effective normalized resistivity as a function of percentage of resistive particles. Experimental data from different authors are compared with the predictions of the Bussian's equation for three values of the cementation exponent $m$. See text for details.

Fig. 3: Schematic view of the two types of simulations: (a) Laboratory measurements, (b) Field measurements. $\mathrm{C}_{1}, \mathrm{C}_{2}$ are the current electrodes injecting $+I$ and $-I$, respectively; $\mathrm{P}_{1}, \mathrm{P}_{2}$ are the potential electrodes.

Fig. 4: Geometry and meshing of the 2D F.E. numerical model simulating field measurements. $a_{\max }$ is the maximum electrode spacing and $\rho_{i}, \rho_{M}, \rho_{e}$ are the resistivities of particles, matrix, and homogeneous external area, respectively. The heterogeneous area (resistivity $\rho_{o}$ ) is $3 a_{\max }$ wide and $0.5 a_{\max }$ thick. A zoom of this area is shown.

Fig. 5: Normalized effective resistivity $\rho_{n}\left(=\rho_{o} / \rho_{M}\right)$ as a function of percentage of circular particles, for a resistivity contrast of 1000 between particles and matrix $\left(\rho_{i} / \rho_{M}=1000\right)$. (a) One size of particles (radius $R$ ). Numerical results for laboratory and field measurement simulations are shown with white and black circles, respectively. They are compared to theoretical formula (full line: Mendelson and Cohen, 1982; dashed line: Bussian, 1983). (b) 
Comparison of the results for three different particle populations (radii $R, R^{\prime}=R / 2$ or $R+R^{\prime}$ in equal proportion).

Fig. 6: Meshing and shape of the three simulated types of particles with an aspect ratio: (a) $A r=1$, (b) $A r=2.5$ and (c) $A r=5$.

Fig. 7: Models with (a) horizontal, (b) inclined and (c) vertical randomly spread particles with an aspect ratio of 2.5 and a particle percentage of $35 \%$.

Fig. 8: Laboratory measurement simulations for a resistivity contrast of 1000 . Normalized effective resistivity $\rho_{n}$ as a function of the particle percentage for three particle orientations: vertical (triangles), inclined at $45^{\circ}$ (diamonds) and horizontal (squares). Results are shown for two different particle aspect ratios: (a) $A r=2.5$; (b) $A r=5$. Results for circular particles are shown with circles. The theoretical equation of Mendelson and Cohen (1982) is drawn for particles longitudinal (L) and transverse (T) to the current lines, and for circular shape (S).

Fig. 9: Field measurement simulations for a resistivity contrast of 1000 . Normalized effective resistivity $\rho_{n}$ as a function of the percentage of particles for three particle orientations: vertical (triangles), inclined at $45^{\circ}$ (diamonds) and horizontal (squares). Results are shown for two different particle aspect ratios: (a) $A r=2.5$; (b) $A r=5$, and fitted with Bussian's equation (dashed line) by adapting the exponent $m$ value. Results for circular particles are shown with circles.

Fig. 10: Normalized average resistivity $\rho_{n}$ as a function of the percentage of particles (with $A r=5$ ) for three values of resistivity contrast: (a) 1000, (b) 100, (c) 10. Numerical results of laboratory measurement simulations are shown for three particle orientations and circular 
particles (same symbols as in Figure 8). The results are fitted with the Bussian's relationship (equation 3), by adapting the $m$ exponent, and with the Mendelson and Cohen formula (equation 2) for a resistivity contrast of 1000 .

Fig. 11: Paradox of anisotropy in a heterogeneous medium containing aligned elongated particles. (a) and (b) Laboratory measurements and associated 2D true resistivity ellipses for vertical and horizontal particles, respectively. (c) and (d) Field measurements and associated 2D apparent resistivity ellipses for vertical and horizontal particles.

Fig. 12: Normalized effective resistivity $\rho_{n}$ (field measurement simulations) as a function of the percentage of elongated particles $(A r=5)$ for a resistivity contrast of 1000 . Numerical results are compared with theoretical results (dotted-dashed lines) taking into account the paradox of anisotropy for two particle orientations: horizontal (squares) and vertical (triangles). Circles and dashed line represent the circular particle case. 